\title{
PAUTAS DE CONSUMO FAMILIAR EN LA CASTILLA PREINDUSTRIAL: PALENCIA, 1750-1850
}

\author{
FERNANDO CARLOS RAMOS PALENCIA * \\ Universidad Carlos III de Madrid
}

\begin{abstract}
RESUMEN
El objetivo primordial de este ensayo es identificar cuáles fueron los determinantes básicos del consumo doméstico de bienes duraderos y semiduraderos en la Castilla del siglo XIX. Hay que distinguir aquellos factores relacionados con variables económicas, es decir, los referidos a la renta familiar y a los elementos que forman parte de ella (salarios, intereses, impuestos, etc.), y los centrados en las propias expectativas sobre la evolución económica, los cambios en la distribución de la renta, las facilidades crediticias o el nivel de inflación, amén de otros de carácter social e institucional. Para ello, abordaremos, en primer lugar, algunos aspectos críticos sobre la fuente utilizada, los inventarios post mortem, y los criterios adoptados en su selección, así como la validez de la muestra obtenida. Por último, realizaremos una aproximación econométrica que permita conocer aquellos elementos que incidían en un mayor o menor consumo familiar.
\end{abstract}

\section{ABSTRACT}

The main objective of this research has been to identify the determinants of durable and semi-durable goods' consumption in Castile between 1750 and 1850. These patterns of family consumption are very different and heterogeneous. It is necessary to distinguish those related with economic variables - family rent and its components (wages and taxes, for example) - and those related to the economic evolution, changes in rent distribution, credit facilities, inflation and other aspects of cultural, social and institutional character. First

* Me gustaría dedicar este artículo, así como agradecer los comentarios y sugerencias realizados, a mi director de tesis Bartolomé Yun Casalilla, asumiendo por mi parte la total responsabilidad sobre los errores cometidos. Asimismo agradezco los comentarios que surgieron en el seminario organizado por la UIMP: «Nuevas interpretaciones en la Historia Económica de España por parte de los comentaristas Ángel García Sanz y Gabriel Tortella y las apreciaciones escritas de Joan R. Rosés y de un evaluador anónimo. Este trabajo se inserta dentro de un proyecto de investigación financiado por la DGICYT «Consumo y Comercialización de textiles en Castilla y en Cantabria: 1750-1914». 
of all, I will explain some critical aspects on the probate inventories, the criteria adopted in their selection and the validity of the sample. An econometric approach that allows to identify the elements that had influenced in the consumption of durable and semi-durable goods has been employed.

\section{LAS FUENTES UTILIZADAS: CRITERIOS DE SELECCIÓN EN LOS INVENTARIOS POST MORTEM}

En esta investigación se han utilizado aproximadamente cuatrocientos inventarios post mortem en tres cortes cronológicos diferentes: 1752-1765, 1785-1800 y 1830-1840; el marco elegido es la provincia de Palencia. El porqué de esta elección tiene que ver con la relativa diversidad económica, a pesar de su carácter marcadamente agrícola, de dicha provincia entre el siglo Xviu y el siglo xIX ${ }^{1}$. Los inventarios post mortem y las escrituras de tasaciones y particiones de bienes realizadas para la adjudicación de herencias recogen la relación completa y detallada de los bienes del difunto ${ }^{2}$. Esto implicaba la tasación de todos los bienes tanto raíces como muebles y semovientes, lo que puede dar una idea aproximada de los distintos niveles de consumo del período considerado. No obstante, la utilización de los inventarios post mortem para el estudio del consumo familiar plantea varios problemas metodológicos cuya extensión sobrepasa el objetivo de este artículo ${ }^{3}$. Dos son, sin embargo, los aspectos sobre los que me gustaría hacer hincapié: en primer lugar, tal y como ha señalado De Vries, los inventarios post mortem reflejan el stock de un determinado producto y no su flujo, es decir, no reflejan los niveles de reposición de los artículos ${ }^{4}$. En segundo lugar, está la representatividad de los inventarios elegidos. Dada la naturaleza de las personas que testaban, casi siempre pertenecientes a las clases sociales más favorecidas, es muy probable que en ocasiones la muestra recogida no sea representativa del nivel de vida y de la riqueza de una población determinada, y se corre el riesgo de obtener una muestra sesgada hacia patrimonios altos y medianos, lo que obliga a seguir una serie de criterios en la selección de los inventarios post mortem ${ }^{5}$.

'Ramos Palencia (1999), pp. 109-113.

2 Para los aspectos legales sobre las herencias en Castilla, véase García (1995), pp. 24-30.

3 Yun (1997), pp. 45.55.

4 De Vries (1993), pp. 85-132.

'En este sentido, Wijsenbeck (1980), pp. 157-176, ha demostrado para los Países Bajos la tendencia hacia patrimonios medianos y altos y la escasez de inventarios cercanos a la base social de cualquier muestra de inventarios post mortem tomada al azar. Y eso 
En principio, se han desechado aquellos inventarios en los que previamente se hubiera realizado un reparto del patrimonio familiar y que hubieran significado una ruptura de la estructura de la familia. Generalmente estos inventarios abundan entre los grupos de edades avanzadas, en los que única y exclusivamente se conservaban aquellos artículos considerados como imprescindibles, o bien se refieren a unidades familiares donde se ha producido con anterioridad la muerte de uno de los cónyuges y una primera división de los bienes (práctica habitual en el régimen sucesorio castellano), con lo que el inventario no responde a lo que podríamos considerar la unidad familiar tipo. Asimismo, se han desestimado aquellos inventarios en los que existía una duda razonable sobre la ocultación de bienes, generalmente de naturaleza urbana y rústica. Una de las principales preocupaciones es garantizar, en la medida de lo posible, un grado de representatividad lo suficientemente amplio en los inventarios elegidos. En este sentido, para reducir el sesgo de la muestra hacia los patrimonios más altos, en el Cuadro 1 se han recogido los ingresos anuales de todos los vecinos que aparecen en el Catastro de Ensenada para distintas localidades palentinas ${ }^{6}$.

Posteriormente, se han computado los ingresos de aquellas familias de quienes se posee el inventario post mortem, y en función del cuerpo general de bienes recogido en dichos inventarios en los tres períodos de tiempo mencionados (1752-1765, 1785-1800 y 1830-1840) se han considerado diferentes intervalos patrimoniales. En concreto, he considerado patrimonios inferiores a 5.000 reales, patrimonios comprendidos entre 5.000 y 10.000 reales, en tercer lugar los patrimonios entre 10.000 y 20.000

incluso en países, como Holanda, donde el inventario se hacía entre los grupos sociales más desfavorecidos como una iniciativa pública que preservara los intereses de los herederos. En esta misma línea argumental, Weatherill (1993), pp. 206-227, ha planteado serias dudas sobre si el cuerpo general de bienes reflejado en el inventario post mortem constituye una medida realmente válida de la riqueza del individuo, y por extensión de su posición social, dada la diversidad existente en las fuentes de ingresos de las economías familiares campesinas.

${ }^{6}$ Dado que los inventarios post mortem proceden de Palencia capital, de la comarca terracampina (Paredes de Nava y Villarramiel) y de distintos pueblos ubicados en las comarcas palentinas (Cerrato, Boedo-Ojeda, Aguilar y Cervera) se han considerado como localidades de referencia o localidades tipo la ciudad de Palencia, los municipios de Tierra de Campos: Paredes de Nava y Villarramiel, y dependiendo de las características de la comarca estudiada (tamaño medio de la población o tipo de actividad económica) las localidades tipo elegidas han sido Villabermudo (para los pueblos ubicados en la comarca situada entre los valles de Boedo y La Ojeda), Villabellaco (para la comarca aguilarense) y Resoba (para la comarca denominada Cervera-Guardo). Los inventarios procedentes del Cerrato tienen como localidad de referencia el municipio de Paredes de Nava dadas las similitudes entre sus estructuras productivas. 


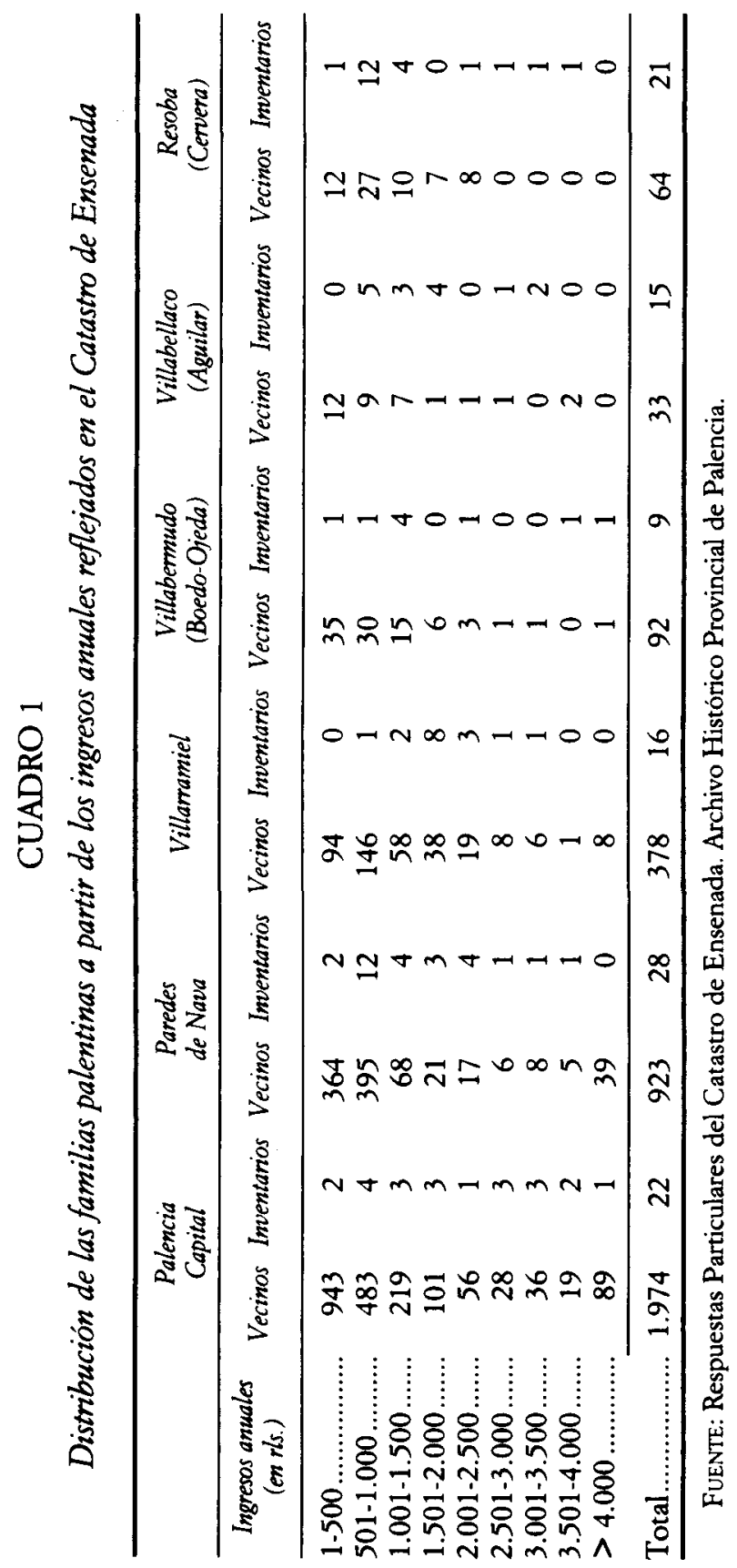


reales, después los que oscilan entre 20.000 y 50.000 reales, en quinto lugar los patrimonios comprendidos entre 50.000 y 90.000 reales, y, finalmente, los superiores a 90.000 reales. Se ha intentado que la mayoria de los inventarios correspondan a patrimonios con una cuantía inferior a los 10.000 reales, los cuales estaban, presumiblemente, más cercanos a la base social de la época y, también probablemente, con unos niveles de ingresos inferiores a los 1.000 reales anuales. Sin embargo, la localización de estos inventarios ha presentado mayores dificultades por razones obvias, ya que normalmente los expedientes o autos judiciales que recogían inventarios o cuentas de partición se realizaban por los problemas que pudieran suscitarse en el reparto de la herencia y, lógicamente, estos problemas no existían cuando no había nada, o muy poco, que repartir. A pesar de estos intentos, en el Gráfico 1 se observa cómo la muestra está relativamente sesgada hacia patrimonios medianos y altos. En dicho gráfico se representa con trazo oscuro la distribución porcentual de las familias palentinas según los ingresos obtenidos en el Catastro de Ensenada, y con trazo claro se indica el porcentaje de los inventarios post mortem disponibles o utilizados entre 1752 y 1765 para cada nivel de ingresos computados en dicho Catastro. En el eje de ordenadas se han recogido los niveles de ingresos con intervalos de 500 reales y en el eje de abcisas el porcentaje de familias que sobre el total hay en cada nivel de ingresos. Se observa que no hay, o no existen, demasiados inventarios que pertenezcan a economías familiares con ingresos inferiores a los 500 reales anuales, a pesar de que gran parte de los inventarios estén por debajo de los 10.000 reales.

En cuanto a las familias con ingresos comprendidos entre 500 y 1.000 reales la muestra conseguida es prácticamente análoga a la reflejada en el Catastro de Ensenada. Para aquellas familias con ingresos anuales comprendidos entre 1.000 y 4.000 reales el porcentaje es siempre superior en la muestra recogida. Estas diferencias en el porcentaje hay que atribuirlas en parte al método de elaboración utilizado en el Catastro. El Catastro de Ensenada para una localidad cualquiera recoge los ingresos que se derivan de las propiedades urbanas o rústicas de esa localidad para todos los vecinos, y no recoge, sin embargo, en las Respuestas Particulares de ese municipio los ingresos que estos mismos vecinos obtienen procedentes de otras propiedades urbanas y rústicas situadas en otra población, los cuales sí figurarian lógicamente en un hipotético inventario de bienes. Es decir, a un vecino de Palencia capital se le computan los ingresos derivados -amén de los que pudiera obtener por su profesión u otras vías- de sus bienes inmuebles ubicados en la capital, pero no de los que pudiera 


\section{GRÁFICO 1}

Distribución porcentual de las familias palentinas a partir de los ingresos anuales reflejados en el Catastro de Ensenada (1749-1753)

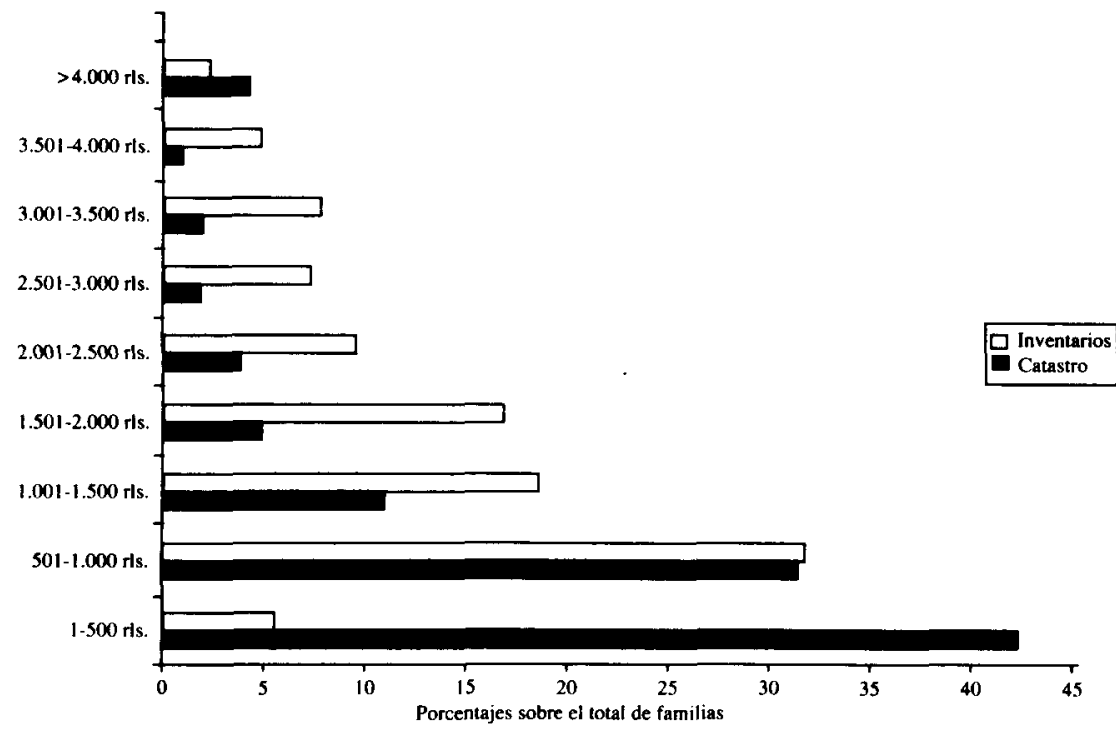

Fuente: Archivo Histórico Provincial de Palencia.

tener en Paredes de Nava, Aguilar de Campóo o cualquier otro lugar. Para conocer la cuantía de estos ingresos habría que acudir a las Respuestas Particulares de estas localidades. Ésta sería una de las razones, además del sesgo inherente a toda muestra tomada aleatoriamente, por las cuales se explicaría que la tendencia (o el porcentaje) de las familias palentinas según los ingresos reflejados en el Catastro de Ensenada esté por debajo de la tendencia de aquellas familias de quienes se conserva el inventario post mortem. Muy probablemente, si se computasen estos ingresos las diferencias serían todavía menores, con lo que la muestra obtenida sería aún más representativa de lo que pudiera pensarse en un primer momento. En resumen, las diferencias se producen fundamentalmente en los extremos, tanto para aquellas familias con ingresos inferiores a 500 reales anuales como para las economías familiares con ingresos superiores a 4.000 reales en un año, lo que puede ponernos sobre la pista de comportamientos muy heterogéneos o, más concretamente, de funciones de demanda muy 
diferentes del consumidor castellano según sea su posición social. En suma, el cruce de los datos de los inventarios con los de las Respuestas Particulares del Catastro de Ensenada ajusta la muestra a una distribución del ingreso muy similar a la que debía existir en 1750 , con lo que se evita el sesgo y el error en que se incurriría si aumentara el tamaño muestral.

Para los cortes temporales de $1785-1800$ y $1830-1840$, teniendo en cuenta que no disponemos de ninguna información suplementaria sobre los ingresos familiares y que la muestra obtenida anteriormente está ligeramente sesgada hacia patrimonios de nivel medio y alto, se han mantenido los mismos intervalos patrimoniales con el fin de reducir dicho sesgo. Quiere esto decir que conforme avancemos en el tiempo la muestra de inventarios post mortem obtenida es cada vez más representativa del nivel de vida existente en cada período y que, además, partimos de un umbral mínimo: entre 1752 y 1765 no existen en la provincia de Palencia inventarios post mortem con un patrimonio o cuerpo general de bienes inferior a los utilizados. La muestra final, una vez aplicados estos criterios, queda recogida en el Cuadro 2.

\section{CUADRO 2}

Distribución de los inventarios post mortem en función del patrimonio

\begin{tabular}{|c|c|c|c|c|c|c|c|c|c|}
\hline \multirow[b]{2}{*}{$\begin{array}{r}\text { Patrimonio } \\
\text { (en reales) }\end{array}$} & \multicolumn{3}{|c|}{$1752-1765$} & \multicolumn{3}{|c|}{$1785-1800$} & \multicolumn{3}{|c|}{$1830-1840$} \\
\hline & Ciudad & Campo & Total & Ciudad & Campo & Total & Ciudad & Campo & Total \\
\hline$<5.000 \ldots \ldots \ldots \ldots$ & 5 & 16 & 21 & 2 & 10 & 12 & 3 & 10 & 13 \\
\hline $5-10.000 \ldots \ldots \ldots \ldots$. & 3 & 22 & 25 & 3 & 24 & 27 & 6 & 24 & 30 \\
\hline $10-20.000 \ldots \ldots \ldots \ldots$ & 4 & 35 & 39 & 6 & 33 & 39 & 5 & 33 & 38 \\
\hline $20-50.000 \ldots \ldots \ldots \ldots$ & 8 & 13 & 21 & 8 & 25 & 33 & 11 & 23 & 34 \\
\hline $50-90.000 \ldots \ldots \ldots \ldots$ & 1 & 2 & 3 & 5 & 5 & 10 & 4 & 5 & 9 \\
\hline$>90.000 \ldots \ldots \ldots \ldots$ & 1 & 1 & 2 & 4 & 4 & 8 & 3 & 5 & 8 \\
\hline Total Inventarios... & 22 & 89 & 111 & 28 & 101 & 129 & 32 & 100 & 132 \\
\hline
\end{tabular}

Fuente: Datos del Archivo Histórico Provincial de Palencia. 


\section{ESTIMACIÓN DE UNA FUNCIÓN DE DEMANDA PARA LAS ECONOMIAS FAMILIARES PREINDUSTRIALES}

Una separación precisa y fiable de los efectos de los factores enunciados anteriormente (riqueza, nivel de ingresos, etc.) sólo puede hacerse con la ayuda de un modelo econométrico bien contrastado que permita calibrar la influencia de cada uno de esos elementos sobre el consumo familiar. Por tanto, el paso siguiente será la especificación econométrica del gasto en consumo -medido a través del stock de bienes duraderos y semiduraderos- en función de la riqueza patrimonial, el volumen de ingresos anuales, el grado de urbanización y el sector económico en que se insertaba la profesión ejercida por el cabeza de familia, a través de una muestra de 370 inventarios post mortem fechados entre 1750 y 1840. Para ello se ha elaborado un modelo econométrico (en cada uno de los tres cortes temporales mencionados: $1752-1765,1785-1800$ y $1830-1840$ ) con todos los datos de corte transversal transformados en logaritmos. El método de estimación propuesto es el de los mínimos cuadrados, computándose los estadísticos " $\mathrm{t}$ " y los errores estándar por el método de White, robusto a la heterocedasticidad ${ }^{7}$. El objetivo primordial consiste en tratar de ave-

${ }^{7}$ Obviando los resultados obtenidos en la utilización de distintas funciones, como la lineal, semi-logarítmica, hipérbola equilátera, log-hipérbola, cuadrática y familia de transformaciones de Box-Cox, he utilizado, dada su flexibilidad, al igual que numerosos trabajos empiricos actuales sobre producción y costes o utilidad y demanda, una función translogarítmica que desarrolla una serie de Taylor de segundo orden alrededor del punto $\ln X=0$, obteniendo:

$$
\ln \mathrm{X} \approx \mathrm{B}_{0}+\sum_{i=1}^{M}\left(\frac{\partial \ln \mathrm{X}}{\partial \ln \mathrm{a}_{i}}\right) \ln \mathrm{a}_{i}+\frac{1}{2} \sum_{i=1}^{M} \sum_{j=1}^{M}\left(\frac{\partial^{2} \ln \mathrm{X}}{\partial \ln \mathrm{a}_{i} \partial \ln \mathrm{a}_{j}}\right) \ln \mathrm{a}_{i} \ln \mathrm{a}_{j}
$$

donde todas las derivadas se evalúan en el punto de expansión. Si identificamos estas derivadas como coeficientes, e imponemos la simetría de las derivadas de "a" cruzadas, la función de X será la siguiente:

$$
\begin{gathered}
\ln \mathrm{X}=\mathrm{B}_{0}+\mathrm{B}_{1} \ln \mathrm{a}_{1}+\ldots+\mathrm{B}_{M} \ln \mathrm{a}_{M}+\delta_{11}\left(\frac{1}{2} \ln ^{2} \mathrm{a}_{1}\right)+\delta_{12} \ln \mathrm{a}_{1} \ln \mathrm{a}_{2}+\delta_{22} \\
\left(\frac{1}{2} \ln ^{2} \mathrm{a}_{2}\right)+\ldots+\delta_{M M}\left(\frac{1}{2} \ln ^{2} \mathrm{a}_{M}\right)
\end{gathered}
$$

Si $\delta_{i j}$ es igual a cero, todo se reduce a una función Cobb-Douglas con una elasticidad de sustitución unitaria constante, que es justamente la utilizada en nuestras investigaciones. Esta función Cobb-Douglas consiste, como se puede apreciar, en una simple transformación doble-logarítmica. Por otra parte, como es bien sabido, la elaboración de modelos econométricos que recoge datos de consumo suscita varios problemas de naturaleza estadística. 
riguar los factores determinantes en el consumo de bienes duraderos y semiduraderos, de tal forma que conociendo la evolución de variables asociadas al tamaño de la población, a la riqueza o al tipo de profesión ejercida se pueda intuir el comportamiento del gasto en bienes duraderos de la economía familiar, del cual, por otra parte, no hay ninguna evidencia estadística ${ }^{8}$.

El referente bibliográfico lo encontramos en Shammas, quien estudia el consumo de bienes duraderos y semiduraderos en las sociedades preindustriales de Inglaterra y de las colonias norteamericanas entre 1550 y 1800, para lo cual utiliza distintas formas funcionales estándar - lineal, semilogarítmica y logarítmica-, concluyendo, a la vista de los $\mathrm{R}^{2}$ obtenidos, que el ajuste logarítmico es el más adecuado a la hora de hacer explícita la relación entre el gasto en bienes de consumo doméstico y la riqueza ${ }^{9}$. Esto implica que conforme aumenta la riqueza, la cantidad dedicada al gasto en bienes duraderos y semiduraderos disminuye, ya que cantidades adicionales de riqueza se destinarían a otros fines. En este sentido, Shammas destaca la mejor adecuación de la curva logarítmica (similar a la que suele atribuirse a los consumidores actuales) con respecto a la curva semi-

Además de la multicolinealidad entre variables dependientes y la autocorrelación, destaca la posible presencia de heterocedasticidad, que puede surgir porque, una vez satisfechas sus necesidades primordiales, una familia de ingresos altos dispone de un mayor excedente de renta que puede ahorrar o gastar en mayor o menor proporción. Ante tal tesitura, el procedimiento más habitual para reducir la heterocedasticidad consiste, asimismo, en la transformación logaritmica de las variables utilizadas. Esto es posible porque dicha transformación comprime las escalas en que se miden tales variables. Asimismo, una ventaja adicional de la transformación logarítmica es que el coeficiente (pendiente) de la variable explicativa mide la elasticidad de la variable exógena con respecto a la variable endógena, es decir, el cambio porcentual en la variable exógena ante un cambio porcentual en el regresor. Véase, por ejemplo, Berndt y Christensen (1973) y Berndt (1990) y más relacionado con el análisis de la demanda Deaton y Muellbauer (1980); Deaton (1983) y Blinder y Deaton (1985).

${ }^{8}$ A mediados del siglo xIx, y tomando como referencia los datos de Le Play (1990), el consumo de bienes duraderos y semiduraderos representa para una familia campesina de Cantabria (1840-47) aproximadamente el 41 por 100, los gastos en alimentación suponen el 51 por 100 mientras que el resto ( 8 por 100 ) se dedicaría al alquiler de vivienda, aseo personal, tabaco y gastos de escritorio. Para una familia de pescadores guipuzcoanos (1856) el gasto en alimentación supone igualmente el 41 por 100, los gastos en bienes duraderos y semiduraderos ascienden al 49 por 100 , mientras que el resto supone el 10 por 100 . Sin embargo, según los datos de Cerdá (1867), la estructura del gasto familiar es bastante diferente para las familias obreras barcelonesas de 1856. Aquí el gasto en alimentación se sitúa en torno al 58 por 100 , el consumo en bienes duraderos supone tan sólo el 20 por 100 , mientras que el resto asciende al 22 por 100 , debido en gran medida al gasto en vivienda que representa el 17 por 100 .

9 Shammas (1990). 
logarítmica (característica del consumidor tradicional) y al ajuste lineal para explicar el consumo en función de la riqueza ${ }^{10}$.

El modo de proceder por mi parte es relativamente sencillo: a partir de la información contenida en el cuerpo general de bienes del inventario post mortem, he efectuado la clasificación de los distintos bienes duraderos y semiduraderos, entendiendo por tales bienes toda clase de ropa, objetos personales, artículos relacionados con el equipamiento del hogar, pinturas, objetos de carácter religioso, libros y objetos de joyería. Obviamente estamos sumando bienes con diferente tasa de depreciación y con distinto tiempo de vida estimado (todo ello sin tener en cuenta apreciaciones subjetivas que tengan relación con el status social y las modas). Para empezar existen notables diferencias dentro de los textiles, así, por ejemplo, dentro de la indumentaria un par de zapatos, pantalones o camisas no tienen la misma duración que la mantelería o la ropa de cama. Idénticas diferencias se producen entre el mobiliario, los instrumentos de cocina o las pinturas ${ }^{11}$. Asimismo, a través del mismo inventario post mortem hemos definido la riqueza patrimonial como la suma de los activos reales, los activos financieros y los bienes de capital o de inversión ${ }^{12}$. Análogo problema al suscitado anteriormente con los bienes duraderos y semiduraderos se produce al estimar la riqueza ${ }^{13}$.

Por otra parte, dados los problemas que presentan los inventarios a la hora de calibrar el peso de las actividades auxiliares en el ingreso de las economías, los datos de cada uno de ellos se han cruzado con los que constan para cada una de esas familias en las Respuestas Particulares

10 Shammas opta por el ajuste logarítmico a tenor de los resultados estadísticos obtenidos (en concreto debido a unos $\mathrm{R}^{2}$ más elevados). En ningún momento menciona la más que segura presencia de heterocedasticidad, hecho que es bastante frecuente en este tipo de modelos econométricos con datos de corte transversal referidos al consumo. Véase en concreto el capitulo 4 de Shammas (1990), pp. 100-118.

"Véase Cerdá (1867), pp. 650-654.

12 Por activos reales considero la suma de propiedades urbanas y rústicas. En cuanto a los activos de carácter financiero, dada la amplia gama de los mismos, he optado por agrupar dentro de esta categoría las siguientes partidas: dinero en metálico, deudas a favor, censos, gastos a cuenta para el funeral, legítimas recibidas por los herederos, etc. Finalmente, por lo que se refiere a los bienes de capital o de inversión he incluido los útiles y herramientas (compuesto por los aperos de labranza, los útiles de bodega y de medición, los útiles para el ganado y los útiles para confeccionar textiles), los bienes perecederos, los textiles sin elaborar y el ganado existente. Por su parte, Shammas (1990), p. 88, distingue entre riqueza $y$ consumo de forma similar.

${ }^{13}$ Véase, por ejemplo, Jorgenson (1990), pp. 19-118, o estimaciones recientes sobre el stock de capital español en Cubel Montesinos y Palafox Gamir (1997), pp. 113-145, y (1998), pp. 619-643. 
del Catastro de Ensenada que, como es sabido, recoge familia por familia y localidad por localidad los ingresos de la población castellana (véase para más detalles el Apéndice, p. 55). Dichos Libros de Respuestas Particulares se elaboraban a partir de las declaraciones de los vecinos seglares y eclesiásticos de cada municipio, atribuyéndose a cada cabeza de familia los datos referentes a su situación personal y económica. Esto, lógicamente, ha supuesto una restricción en el volumen de la muestra a considerar; pero ha permitido valorar la influencia de los ingresos anuales de las economías familiares en el consumo doméstico, hecho que, por otra parte, supone una auténtica novedad dentro de la historiografía económica.

Otra de las variables considerada ha sido el grado de urbanización, que puede orientarnos sobre el grado de accesibilidad a los mercados y las diferentes pautas de demanda rurales o urbanas en el gasto de bienes duraderos y semiduraderos. Estadísticamente se ha considerado la urbanización como una variable ficticia que toma el valor 1 si la familia vivía en la capital palentina y el valor 0 si vivía en cualquier otra localidad de la provincia ${ }^{14}$. La última variable considerada ha sido la ocupación profesional o actividad económica. En este sentido es interesante realizar una aproximación a la posible influencia que la dedicación preferente a las distintas actividades económicas (primaria, secundaria y terciaria) pudo haber tenido sobre los patrones de consumo. Por ello, y pasando por alto las dificultades de clasificación de la población preindustrial por sectores económicos, esta última variable se incluye de nuevo como una variable ficticia, que toma el valor 1 para los individuos que trabajan en el sector primario y el valor 0 en los demás casos. La duda surge en el caso de

${ }^{14}$ El principal problema que surge al analizar el proceso de desarrollo urbano es el criterio adoptado para definir población urbana y población rural. Según Bairoch y Goertz (1986), el factor más relevante sería el tamaño de las poblaciones; en este sentido, en la Europa del siglo XIX, el tamaño mínimo a partir del cual un municipio era considerado como ciudad oscilaba entre los 2.000 y los 10.000 habitantes. No obstante, en determinadas ocasiones se han sugerido otros criterios como la proporción de población activa ocupada en los sectores secundario y terciario, Duncan (1974), pp. 191-217, o la densidad de población, De Vries (1987). Teniendo en cuenta estas consideraciones, Camps (1995) utiliza el umbral mínimo de los 2.000 habitantes cuando estudia la urbanización catalana entre los siglos xviı y XIX y analiza la formación del mercado de trabajo industrial en la Cataluña del siglo XIX. En el caso que nos ocupa, la distinción entre rural y urbano se ha ceñido única y exclusivamente en la denominación de ciudad. Podrían, por tanto, surgir dudas con respecto a dos de las localidades analizadas, en concreto, Paredes de Nava y Villarramiel. Sin embargo, si comparamos el desarrollo de los sectores secundario y terciario de ambas localidades con otros municipios del territorio español (en particular con los catalanes), creo que esta distinción refleja las principales diferencias entre población rural y municipios con infraestructura urbana. 
aquellas economías familiares que perciban ingresos procedentes de ambos sectores. En este caso, la adscripción a uno u otro sector productivo ha estado en función de la cuantía de los ingresos computados en el Catastro de Ensenada. Por ejemplo, si los ingresos procedentes de la agricultura o la ganadería eran superiores a los provenientes de actividades industriales o artesanales, automáticamente la economía familiar ha sido adscrita al sector primario y viceversa.

Los resultados obtenidos en el Cuadro 3 son muy expresivos. En los tres cortes cronológicos especificados (1752-1765, 1785-1800 y 1830-1840) todos los regresores resultan significativos con $t$-student mayores que 2 . Asimismo, se obtiene en todas las estimaciones un $R^{2}$ próximo a 0,7 . Esto significaría que aproximadamente el 70 por 100 del gasto en consumo de bienes duraderos y semiduraderos vendría explicado por el valor del patrimonio familiar, por el grado de urbanización existente y por el sector económico en el cual se insertaba la profesión o actividad predominante ejercida por el cabeza de familia.

En la estimación de estas funciones de demanda para las economías preindustriales sobresale la importancia de la riqueza patrimonial. En este sentido, si la riqueza variase en un 1 por 100 el consumo familiar aumentaría entre un 0,63 y un 0,53 por 100 aproximadamente entre $1750-1840$, tal y como indican las elasticidades de la renta sobre el consumo. ¿Qué ocurre con el resto de la renta que no se gasta? Casi con toda seguridad, la canalización de esa renta sobrante tuvo que haberse dirigido hacia el consumo de bienes perecederos - altamente condicionado por las fluctuaciones de los precios agrícolas- y de bienes fungibles, aunque tampoco debiera desdeñarse en este contexto la capacidad de ahorro que tenían estas economías familiares.

Asimismo, destaca el peso de la urbanización en la potenciación de la demanda y la significación de los centros de comercialización que representan las ciudades ${ }^{15}$. Esto refuerza el grado de importancia de los núcleos de mayor población en la difusión del consumo doméstico debido, sobre todo, a la mayor facilidad de acceso a los mercados y, también, a que se acrecientan las posibilidades de emulación e imitación de diferentes modelos de consumo entre los distintos grupos sociales en aquellas localidades con más habitantes y con un mayor dinamismo social y económico.

15 Sorprende un valor superior a la unidad entre 1752.65 (1.033), pero cabría recordar que cuando introducimos variables ficticias no estamos midiendo elasticidades. Véase Halvoresen y Palmquist (1980), pp. 474-475. 


\section{CUADRO 3}

Ecuaciones estimadas sobre el consumo familiar entre 1750 y 1840. (Variable dependiente en logaritmos: consumo doméstico en reales)

\begin{tabular}{|c|c|c|c|c|}
\hline Variables explicativas en logaritmos & $1752-1765^{*}$ & $1752-1765$ & $1785-1800$ & $1830-1840$ \\
\hline Riqueza patrimonial. & $\begin{array}{c}0,467 \\
(7,194)\end{array}$ & $\begin{array}{c}0,631 \\
(10,948)\end{array}$ & $\begin{array}{c}0,536 \\
(8,895)\end{array}$ & $\begin{array}{c}0,578 \\
(13,409)\end{array}$ \\
\hline Volumen de ingresos anuales.......... & $\begin{array}{c}0,357 \\
(4,013)\end{array}$ & - & - & - \\
\hline Grado de urbanización ................... & $\begin{array}{c}0,970 \\
(8,516)\end{array}$ & $\begin{array}{c}1,033 \\
(8,484)\end{array}$ & $\begin{array}{c}0,802 \\
(5,549)\end{array}$ & $\begin{array}{c}0,713 \\
(5,818)\end{array}$ \\
\hline Profesión del cabeza de familia ..... & $\begin{array}{c}-0,446 \\
(-3,814)\end{array}$ & $\begin{array}{c}-0,487 \\
(-4,032)\end{array}$ & $\begin{array}{c}-0,413 \\
(-3,627)\end{array}$ & $\begin{array}{c}-0,623 \\
(-6,766)\end{array}$ \\
\hline Constante.. & $\begin{array}{c}-0,093 \\
(-0,149)\end{array}$ & $\begin{array}{c}0,968 \\
(1,778)\end{array}$ & $\begin{array}{c}2,082 \\
(3,384)\end{array}$ & $\begin{array}{c}1,862 \\
(4,531)\end{array}$ \\
\hline $\mathrm{R}^{2}$. & 0,707 & 0,679 & 0,650 & 0,740 \\
\hline $\mathrm{R}^{2}$ ajustado ..................... & 0,696 & 0,670 & 0,642 & 0,734 \\
\hline Núm. observaciones....................... & 111 & 111 & 128 & 131 \\
\hline 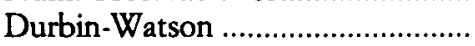 & 1,843 & 1,970 & 2,079 & 2,030 \\
\hline Estadístico F................ & 64,10 & 75,51 & 76,91 & 120,65 \\
\hline
\end{tabular}

Notas:

* Incluye los ingresos anuales como variable explicativa.

Definición de variables:

Ficticia Grado de Urbanización: valores unitarios si el individuo vive en Palencia capital. Cero en los demás casos.

Ficticia Profesión del Cabeza de Familia: valores unitarios para los individuos que trabajan en el sector primario. Cero en los demás casos.

Las cifras entre paréntesis corresponden a los valores de la t-student.

También resulta interesante el comportamiento de la variable relacionada con la actividad económica ejercida por el cabeza de familia. Como observamos, su coeficiente siempre es negativo $(-0,487,-0,413$ y $-0,623)$, lo que implica una relación negativa entre aquellos individuos que trabajaban en el sector primario y la posesión de bienes duraderos y semiduraderos. Es decir, aquellos individuos que se dedicaban a actividades relacionadas con la industria, la artesanía o el comercio tenían una mayor propensión al consumo de bienes duraderos y semiduraderos, lo que corrobora las conclusiones de otros estudios sobre el tema ${ }^{16}$.

${ }^{16}$ Aunque con ciertos matices, Shammas (1990), pp. 104-111, llega a conclusiones similares. Dicha correlación negativa únicamente alcanza resultados satisfactorios en Wor- 
Veamos qué sucede cuando incluimos entre 1752-1765 al volumen de ingresos anuales como variable explicativa, ya que, desafortunadamente, para los años comprendidos entre 1785-1800 y 1830-1840 no disponemos de ninguna fuente fiscal que recoja los ingresos. En principio, si el nivel de ingresos anuales variase en un 1 por 100 la demanda de las familias aumentaría en un 0,357 por 100 , porcentaje inferior al que se produciría si se modificase el nivel de riqueza ${ }^{17}$. Las explicaciones pueden ser dos. Por un lado, el grado de correlación entre dos variables stock (consumo familiar-riqueza patrimonial) es más alto que la correlación entre una variable flujo y una variable stock (volumen de ingresos anuales-consumo familiar) ${ }^{18}$. Por otro lado, el hecho de que la elasticidad de la demanda del consumo doméstico en función de la riqueza sea superior a la elasticidad de demanda en función de los ingresos anuales tiene connotaciones muy interesantes desde la teoría económica. Para el pensamiento keynesiano la renta disponible es el principal determinante del consumo y, por tanto, cualquier otra variable - riqueza o tipos de interés - no desempeña un papel importante. Ocurre, sin embargo, que los consumidores intentan maximizar su utilidad asignando un flujo de ingresos de toda la vida a un patrón óptimo vitalicio de consumo. En otras palabras, cuando las familias se enfrentan a la decisión de cuánto consumir hoy y cuánto consumir mañana, tienen en cuenta las rentas que esperan obtener en el futuro y el consumo de bienes y servicios que con dichas rentas serán capaces de adquirir. Por consiguiente, el papel de la renta disponible en la determinación del consumo corriente tiende a reducirse y el de la riqueza - los activos reales y financieros poseídos por los individuos - a aumentar. En este contexto se han formulado dos teorías alternativas a la función de consumo keynesiana: la hipótesis del Ciclo Vital y la teoría de la Renta Permanente ${ }^{19}$. La hipótesis del ciclo vital formulada por Modigliani y Ando pone especial énfasis en dos aspectos: la renta varía a lo largo de la vida

cestershire entre 1670 y 1720 . En ninguna otra parte de Inglaterra o de sus colonias en Norteamérica se vuelve a producir dicha correlación. Por su parte, Weatherill (1988) también encuentra una cierta relación entre el menor consumo de bienes duraderos y la dedicación a tareas agrícolas.

${ }^{17}$ La riqueza patrimonial y el volumen de ingresos anuales suelen estar correlacionados, ya que normalmente los individuos con mayor riqueza son quienes generalmente también poseen mayores ingresos. En este caso el grado de correlación se sitúa en 0,67.

${ }_{18}$ Aunque los datos procedentes del consumo familiar y de la riqueza procedan de la misma fuente (inventarios post mortem) no podemos hablar de multicolinealidad o colinealidad entre las variables, ya que conceptualmente ésta sólo puede darse entre variables explicativas.

${ }^{19}$ Véase Modigliani y Ando (1963), pp. 55-84, y Friedman (1957), respectivamente. 
de los consumidores y el ahorro permite a los individuos desplazar su renta desde los períodos en que es más alta a aquellos en que es más baja. Esto quiere decir que la teoría del ciclo vital determina el consumo como una función de la renta esperada, que se divide en las rentas procedentes del trabajo y las derivadas de la riqueza. Por su parte, Friedman divide la renta corriente en renta permanente (aquella que los individuos piensan mantener en el futuro: posesión de activos reales y financieros y dotación de capital humano) y en renta transitoria (aquella que depende de factores coyunturales). El ingreso permanente se configura así como el determinante fundamental del consumo y, por tanto, éste depende de la proporción de la renta que una persona espera mantener el resto de su vida, dado su nivel actual de riqueza y su renta actual y futura. En definitiva, según estas dos teorías las economías familiares actuales planifican su comportamiento respecto al consumo y al ahorro en horizontes temporales amplios, con la intención de maximizar su función de utilidad intertemporal sujeta a una restricción presupuestaria.

A partir de estas premisas, ¿cuáles son las conclusiones que se pueden extraer de los resultados de nuestra estimación? En principio, dados los valores obtenidos, podemos afirmar que las familias castellanas de mediados del siglo Xvil adquieren nuevos bienes de carácter duradero o semiduradero teniendo más en cuenta su renta permanente (riqueza reflejada en los inventarios post mortem) que su renta disponible (ingresos computados en el Catastro de Ensenada), es decir, en la línea argumentada por Modigliani y Ando o Friedman. En suma, conviene enfatizar el papel desempeñado por el volumen de ingresos anuales, no sólo por sus resultados econométricos, sino por la novedad que constituye dentro de la historiografía económica internacional, ya que normalmente se ponía en relación el gasto en consumo con la riqueza o el patrimonio reflejado en el cuerpo general de bienes. Además la estimación de los ingresos no se circunscribe a la mera observación de las rentas salariales, puesto que éstas -quizás las que pudieran constituir una estimación más arbitraria dentro del Catastroson sólo una parte del ingreso total, lo que disminuye el margen de error en la aplicación del modelo econométrico.

Para calibrar la importancia de este modelo, los resultados de esta estimación se pueden comparar con los obtenidos por Shammas a partir de los inventarios post mortem procedentes de Inglaterra y de sus colonias norteamericanas. El modelo logarítmico de Shammas incluye como factores explicativos del consumo de bienes duraderos la riqueza, el tamaño de la economía familiar, la residencia o no en ciudad y diferentes status 
socio-profesionales (en concreto elite, farmer, tradesman y widow, e incluso los book owner para tratar de valorar los efectos de la educación en el consumo) ${ }^{20}$. Los resultados de esta estimación señalan a la riqueza, básicamente, y, en menor medida, al tamaño de la economía familiar como los principales determinantes del consumo familiar. El resto de las variables, dependiendo del lugar y del período, resultan significativas en contadas ocasiones. Con respecto a los $\mathrm{R}^{2}$, éstos son mayores para las colonias norteamericanas, en torno al 76 por 100, que para la sociedad inglesa, ligeramente superiores al 70 por 100 . Por su parte, para la provincia de Palencia, además del peso de la riqueza y de los ingresos anuales familiares (una variable ausente en estudios anteriores), el resto de las variables explicativas utilizadas, similares a las de Shammas (el grado de urbanización y la profesión del cabeza de familia) son bastante más relevantes en la sociedad castellana que en la sociedad inglesa. En cuanto a la valoración final del modelo, se obtiene un $\mathrm{R}^{2}$ próximo al 70 por 100 , no muy dispar de los resultados obtenidos para la sociedad inglesa y la de sus colonias norteamericanas.

Para finalizar, hay que hacer hincapié en la importancia de los resultados obtenidos y en su extrapolación para el estudio de otras sociedades. El modelo econométrico especificado explica más de tres cuartas partes del gasto en bienes duraderos y semiduraderos, quedando únicamente pendiente de cuantificar aquellos juicios de valor, no por ello menos importantes, que se realizan en función de las expectativas futuras sobre la evolución política, económica o institucional o la influencia de las modas, aspectos todos ellos de difícil valoración.

20 Shammas (1990), pp. 104-111. En esta línea, he intentado utilizar también como variables explicativas adicionales el gasto superior a los 10 reales en libros. Se ha computado, lógicamente, como una variable ficticia asignando 1 a aquellas familias con libros y 0 en los demás casos. Dicha variable resultó significativa para los dos últimos cortes temporales considerados: $1785-1800$ y 1830-1840. Sin embargo, existía una fuerte correlación entre esta variable $y$ el volumen de riqueza computado, de tal forma que para evitar posibles problemas de multicolinealidad la he omitido en el análisis final. En cuanto a la consideración de la variable viuda (en la muestra final aparecen 29 inventarios de viudas sobre los 370 totales), al igual que Shammas, no era significativa en ninguno de los tres períodos. Finalmente, también deseché la variable relacionada con el tamaño de la economía familiar (Shammas obtiene dicho dato multiplicando el número de camas existentes por 2 y computando 0,5 si no aparece cama alguna para evitar problemas a la hora de tomar logaritmos en las variables), ya que en muchos de los inventarios no se indica, con absoluta certeza, quienes conviven en el hogar familiar. Por otra parte, la metodología planteada por Shammas suscita problemas con otro tipo de objetos utilizados para dormir (como, por ejemplo, los jergones o los bancos), así como serias dudas en el coeficiente utilizado. 


\section{CONCLUSIONES}

Un análisis global sobre los coeficientes de las variables explicativas permite señalar las siguientes conclusiones. En primer lugar, la elasticidad de demanda del consumo de bienes duraderos y semiduraderos en función de la riqueza (elasticidad renta de la demanda) es relativamente estable, ya que oscila entre el 63 y el 53 por 100 entre 1750 y 1840. Es decir, por cada unidad adicional de riqueza la proporción invertida en bienes duraderos y semiduraderos fue más o menos similar. Por otra parte, el hecho de que la elasticidad de la demanda del consumo doméstico en función de la riqueza sea superior a la elasticidad de demanda en función de los ingresos anuales entre 1752-1765 implica que las familias castellanas supeditaban su consumo más a su renta permanente (riqueza reflejada en los inventarios post mortem) que a su renta disponible (ingresos computados en el Catastro de Ensenada).

Asimismo destaca la paulatina pérdida de importancia del grado de urbanización como factor condicionante del consumo de bienes duraderos y semiduraderos. El motivo es evidente y no es otro que la disminución de las diferencias socio-económicas entre Palencia y los núcleos rurales de mayor entidad, lo que, por otra parte, está íntimamente ligado al proceso de integración de los mercados en una economía con un proceso de urbanización lento ${ }^{21}$. La cuestión a resolver es si la disminución de estas diferencias entre Palencia y otros núcleos rurales se debió a un aumento de la actividad económica de estos últimos, o si, por el contrario, se debió a la pérdida de peso específico de la capital ${ }^{22}$. Finalmente, la profesión del cabeza de familia adquiere especial relevancia entre 1830 y 1840 , síntoma evidente de que las rentas generadas por los sectores secundario y terciario son cada vez más importantes que las generadas por la agricultura. Por tanto, conforme se van produciendo los procesos de integración de los mercados, la distinción entre ciudad y campo pierde valor a la hora

${ }^{21}$ Castilla la Vieja y León tiene, junto con Aragón, uno de los índices más bajos de crecimiento en las tasas de urbanización. Reher (1990), p. 43.

${ }_{22}$ El hecho de que la población de la ciudad apenas aumentara entre 1759 (9.639 habitantes) y 1850 (11.480 habitantes) y el número de telares, a juzgar por las noticias de Larruga (t. XXXIII, pp. 37-223), y sus cifras de producción no experimentaran avances hasta finales del siglo xvil y que, mientras tanto, otras localidades cercanas como Paredes de Nava o Villarramiel duplicasen su población en las mismas fechas constituye un claro indicativo de la competitividad de la industria rural textil. En el futuro, las crisis políticas, institucionales y económicas de principios del siglo xIX que habían alterado la estructura social y productiva de la provincia precipitaron la desindustrialización del entorno rural y de la capital. Véase García Colmenares (1992). 
de interpretar el gasto en bienes de consumo duradero y semiduradero, configurándose la riqueza, básicamente, y el grado de desarrollo comercial $e$ industrial como las variables más influyentes en el consumo doméstico.

En suma, podemos afirmar, a tenor de los resultados obtenidos, que la conexión causal entre el consumo de bienes duraderos y semiduraderos (medido a partir del stock inventariado de dichos productos) y la riqueza patrimonial (nivel de renta en un sentido amplio del concepto), más el volumen de ingresos anuales, el grado de urbanización y el tipo de actividad económica ejercida por el cabeza de familia, es bastante explícita entre 1750 y 1850 , lo que constituye una notable mejora sobre el modelo desarrollado por Shammas ${ }^{23}$.

Queda por contrastar definitivamente si hubo o no un incremento del consumo familiar de bienes duraderos y semiduraderos. En este sentido y considerando que los coeficientes correspondientes a la riqueza y a la profesión del cabeza de familia (las dos variables que toman paulatinamente más importancia en la decisión final de los consumidores castellanos) se incrementaron desde finales del siglo xVII, los resultados obtenidos apoyarían la idea de que se produjo un aumento gradual del consumo doméstico, lo que lleva a revisar la idea demasiado pesimista del consumidor castellano y toda una serie de suposiciones sobre lo que pudo ser su evolución durante el siglo $\mathrm{XIx}^{24}$. No obstante, es evidente que no estábamos ante una sociedad inmovilista, ya que un sector relativamente amplio de la población castellana (ejemplificado en la provincia palentina) era sensible a los cambios en sus niveles de riqueza o en sus ingresos e incluso hacia el grado de apertura de las economías locales. En suma, reaccionaba ante los estímulos provenientes del mercado, lo cual traslada nuestras reflexiones

${ }^{23}$ Asimismo, un análisis menos formalizado estadísticamente y más tradicional revela igualmente conclusiones similares. Ramos Palencia (2000), pp. 15-23.

${ }^{24}$ En este sentido, conviene recordar que desconocemos el ritmo de reposición de los bienes duraderos y semiduraderos - aunque hay razones para suponer que se aceleró en el siglo XIX al mismo tiempo que se daba una reducción del consumo de productos textiles de segunda mano- y, además, el mayor o menor porcentaje de gasto en este tipo de bienes puede ser reflejo de cambios en la estructura de precios relativos que hayan conducido a una revalorización de determinados bienes comprendidos en el inventario, como, por ejemplo, las propiedades rústicas, con lo que la proporción del stock de bienes duraderos y semiduraderos sobre el valor total de éstos puede haberse mantenido estable incluso en el caso de que se haya producido un aumento de su consumo. Asimismo, cabe considerar que quizás estemos ante un descenso del precio relativo de este tipo de bienes de consumo, lo que, por otra parte, sería coherente con el desarrollo, por ejemplo, del sector textil durante las primeras fases de la industrialización. Es decir, se estaría consumiendo una mayor cantidad de bienes duraderos y semiduraderos, aunque el porcentaje de gasto que representan en el inventario post mortem no variase. 
al lado de la oferta y la distribución. En este sentido, existen evidencias donde se aprecia que la cantidad de tiendas especializadas en productos diferentes a los alimenticios en las áreas rurales fue pequeña, y no varió mucho respecto de la situación de $1750^{25}$. Sin embargo, es necesaria una perspectiva autocrítica, ya que aunque existen signos evidentes de un comportamiento del consumidor preindustrial distinto al que pensábamos, no es menos cierto que en la sociedad del Antiguo Régimen con, previsiblemente, funciones de demanda tan dispares en sus extremos, el ulterior desarrollo económico vino acompañado por una progresiva convergencia de las funciones de demanda de los distintos grupos sociales amén del desarrollo creciente de las actividades industriales y comerciales y de los procesos de urbanización.

\section{APÉNDICE \\ Ingresos Computados en el Catastro de Ensenada}

\section{Ingresos procedentes de las propiedades urbanas}

Se descuentan o deducen aquellas cargas o censos que existan sobre dichas propiedades.

\section{Ingresos procedentes de las propiedades rústicas}

Se descuentan o deducen aquellas cargas o censos que existan sobre dichas propiedades.

\section{Ingresos relacionados con la ganadería}

Se refiere a los ingresos estipulados por la posesión de ganado y/o a la realización de actividades en aparcería.

\section{Censos a favor}

\section{Ingresos (utilidad) procedentes del trabajo personal $u$ oficio}

El Catastro de Ensenada computa una renta denominada personal a aquellos varones que tengan un oficio y sean menores de sesenta años. Las profesiones liberales, como, por ejemplo, médico, alguacil o mercader, tienen una utilidad de 2.000, 3.000 reales, etc., según los casos. En el caso de que el cabeza de familia se dedique al sector secundario o terciario

\footnotetext{
25 Yun (1994)
} 
se computan ciento ochenta días variando el jornal diario hasta un máximo de 7 reales (es el caso de los maestros tallistas o carpinteros). Si por contra se dedica al sector primario se computan ciento veinte días. Estos últimos ingresos oscilan en la provincia palentina entre 2 reales para las zonas de montaña y menos fértiles y 4 reales y 17 maravedíes para aquellos lugares más productivos, generalmente situados en Tierra de Campos. Así mismo, dentro del sector primario aquellos que se dedicaban al pastoreo se les computaban 360 días cuyo jornal diario también varía en función de la ubicación del municipio.

\section{Ingresos derivados de actividades complementarias o auxiliares}

a) Ingresos derivados del trabajo personal $u$ oficio

Generalmente se refiere al comercio de telas, pieles, ventas de lienzos («industria del lino»), estameñas o paños en aquellas profesiones relacionadas con el sector textil y a los ingresos que obtienen los labradores por llevar (arrendatarios) otras tierras que no son de su propiedad.

b) Ingresos procedentes de actividades agricolas

Dado el carácter marcadamente agrícola de la economía castellana en el siglo xviI raro era el caso en que una familia no se dedicase a este tipo de tareas.

\section{c) Otros ingresos}

Estos ingresos procedían de diferentes actividades, siendo las más habituales la recogida de leña y hoja, la recogida de piedra, la realización de horas en el molino de la localidad y la fabricación de cubas.

\section{Notas:}

1. A las personas mayores de sesenta años y a las mujeres viudas o solteras el Catastro de Ensenada no les computa renta alguna procedente del trabajo personal.

2. En el caso de que algún hijo mayor de catorce años o criado realizase cualquier tipo de actividad remunerada, estos ingresos se computan como ingresos complementarios o auxiliares.

\section{BIBLIOGRAFIA}

Bairoch, P., y Goertz, G. (1986): «Factors of Urbanization in the Nineteenth Century Developed Countries: A Descriptive and Econometric Análisis», Urban Studies, 23, 4, pp. 285-305. 
Berndt, E. (1990): The Practice of Econometrics, Reading, Mass., Addison-Wesley. Berndt, E., y Christensen, L. (1973): «The Translog Function and the Substitution of Equipment, Structures, and Labour in U.S. Manufacturing, 1929-1968», Joumal of Econometrics, 1, 1, pp. 81-114.

Blinder, A., y Deaton, A. (1985): «The Time Series Consumption Function Revisited», Brookings Papers on Economic Activity, 2, pp. 465-521.

BREWER, J., y PORTER, R. (eds.) (1993): Consumption and the World of Goods, London-New York, Routledge.

CAMPS I CURA, E. (1995): La formación del mercado de trabajo industrial en la Cataluña del siglo XIX, Madrid, Ministerio de Trabajo y Seguridad Social.

CERDÁ, I. (1867, 1968): «Monografía estadística de la clase obrera de Barcelona en 1856», Teoria General de la Urbanización y aplicación de sus principios $y$ doctrinas a la Reforma y Ensanche de Barcelona, 3 vols., Madrid, Instituto de Estudios Fiscales.

Cubel Montesinos, A., y Palafox Gamir, J. (1997): «El stock de capital de la economía española, 1900-1958», Revista de Historia Industrial, 12, pp. 113-145.

- (1998): «La continuidad del crecimiento económico en España, 1850-1936», Revista de Historia Económica, 16, 3, pp. 619-643.

DeATon, A. (1983): «Demand Analysis», en Z. Griliches y M. INTRILIGATOR (eds.), Handbook of Econometrics, Amsterdam, North Holland.

Deaton, A., y Muellbauer, J. (1980): Economics and Consumer Bebavior, New York, Cambridge University Press.

Duncan, O. D. (1974): «Population Distribution and Community Structure», en C. Tilly (ed.), An Urban World, Boston-Toronto, pp. 191-217.

Friedman, M. (1957): Theory of Consumption Function, Princeton, Princeton University Press.

García, M. (1995): Herencia y Patrimonio Familiar en la Castilla del Antiguo Régimen (1650-1834), Valladolid, Universidad de Valladolid.

GaRcia Colmenares, P. (1992): Evolución y crisis de la industria textil castellana. Palencia, 1750-1990, Madrid, Mediterráneo.

Halvorsen, R., y Palmouist, R. (1980): «The Interpretation of Dummy Variables in Semilogarithmic Equations», American Economic Review, 70, 3, pp. 474-475.

JORGENSON, D. (1990): «Productivity and Economic Growth», en E. R. BERNDT, y J. E. TRLPLETT (eds.), Fifty Years of Economic Measurement, Chicago, University Chicago Press, pp. 19-118.

LARRUGa, E. (1787-1794): Memorias politicas y económicas sobre los frutos, fábricas, comercio y minas de España, tt. XXXII y XXXIII, Madrid.

LE PLAY, F. (1990): Campesinos y pescadores en el Norte de España: tres monografias de familias trabajadoras a mediados del siglo XIX, Madrid, Ministerio de Agricultura, Pesca y Alimentación.

Modigliani, F., y ANDO, A. (1963): «The Life-Cycle. Hypothesis of Saving: Aggregate Implications and Test», American Economic Review, 53, 1, pp. 55-84.

Ramos Palencla, F. C. (1999): «Una primera aproximación al consumo en el mundo rural castellano a través de los inventarios post mortem: Palencia, 1750-1840», en J. Torras y B. Yun (eds.), Consumo, condiciones de vida y comercialización en Cataluña y Castilla, ss. XVII.XIX, Valladolid, Junta de Castilla y León, Consejería de Educación y Cultura, pp. 107-131. 
- (2000): «El consumo familiar de bienes duraderos y semiduraderos en la Castilla Pre-industrial: Palencia, 1750-1850». Documento de trabajo, serie de Historia Económica e Instituciones 00-05, Madrid, Universidad Carlos III.

ReHer, D. S. (1990): Town and Country in pre-industrial Spain. Cuenca, 1550-1870, Cambridge, Cambridge University Press.

Shammas, C. (1990): The Pre-industrial Consumer in England and America, Oxford, Clarendon Press.

Schudrman, A., y Walsh, L. (eds.) (1994): Material Culture: Consumption, Life-Style, Standard of Living, 1500-1900, Actas de «XI Conferencia Internacional de Historia Económica», Milano, Universitá Boconni.

TORRAS, J., y Yun, B. (eds.) (1999): Consumo, condiciones de vida y comercialización en Cataluña y Castilla, ss. XVII-XIX, Valladolid, Junta de Castilla y León: Consejería de Educación y Cultura.

DE VRIES, J. (1987): La urbanización en Europa 1500-1800, Barcelona, Crítica.

- (1993): «Between Purchasing Power and the World of Goods: Understanding the Household Economy in Early Modern Europe», en J. BREWER y R. PORTER (eds.): Consumption and the World of Goods, London-New York, Routledge, pp. 85-132.

WEATHERILL, L. (1988): Consumer Behaviour and Material Culture in Britain, 1660-1760, London-New York, Routledge.

- (1993): «The Meaning of Consumer Behaviour in Late Seventeenth - and Early Eighteenth- Century England», en J. Brewer y R. PORTER (eds.), Consumption and the World of Goods, London-New York, Routledge, pp. 206-227.

WIJSENBECK, T. (1980): «Delf in the Eighteenth Century», en H. VAN DER WOUDE y A. Schuurman (eds.), Probate Inventories. A New Source for the Historical Study of Wealth, Material Culture and Agricultural Development, Leewenborch Conference (Wageningen, 5-7 mayo), Utrecht, pp. 157-176.

Yun, B. (1994): «Peasant Material Culture in Castile (1750-1900): Some Proposals», en A. J. SchuURman y L. WaLSH (eds.), Material Culture: Consumption, Life-Style, Standard of Living, 1500-1900, Actas de «XI Conferencia Internacional de Historia Económica», Milano, Universitá Boconni, pp. 125-136.

- (1997): «Inventarios post mortem, consumo y niveles de vida del campesinado del Antiguo Régimen. (Problemas metodológicos a la luz de la investigación internacional», en VIII Congreso de Historia Agraria. Preactas, Salamanca, 28-30 de mayo de 1997), pp. 45-55.

\section{FUENTES DOCUMENTALES}

Archivo Histórico Provincial de Palencia (AHPP):

Sección Protocolos Notariales:

Años 1752-1765:

Legajos 97, 98, 99, 379, 433, 434, 435, 642, 643, 675, 1000, 2656, 2733, 3731, $3732,3793,3795,6277,6278,6962,6964,7814,7816,7817,10617,10618$, $10619,10834,11383$ y 11384. 
Años 1785-1800:

Legajos $107,522,523,524,525,628,629,630,653,700,731,3065,3066,3165$, $3354,3356,6075,6076,6078,6082,6537,6538,6539,6540,6541,6542$, $7226,7343,7347,7461,7462,7463,7513,7515,7519,7733,7734,10605$, 11358 y 11359.

Años 1830-1840:

Legajos $360,361,376,377,378,547,1194,1195,1238,1239,3491,3493,3494$, $3495,3496,4440,6490,7256,7537,7538,7540,11987,11988,12847,12863$, $12869,12870,12871,12877$ y 12878.

Catastro de Ensenada: Respuestas Generales y Particulares. 